\title{
NOTE ON SUBNORMAL WEIGHTED SHIFTS
}

\author{
PENG FAN
}

(Communicated by John B. Conway)

\begin{abstract}
The purpose of this note is two-fold: (1) To point out that Stampfli's characterization of subnormal shifts can be reformulated in operator form which turns out to be the Spitkovskii's characterization of subnormal operators; (2) To use this reformulation to give a new proof of the subnormality of a class of shifts.
\end{abstract}

In [5], Stampfli explicitly exhibited for a subnormal shift $F_{1}$ its minimal normal extension

$$
N=\left[\begin{array}{cccc}
F_{1} & G_{2} & & 0 \\
& F_{2} & G_{3} & \\
& & F_{3} & \ddots \\
0 & & & \ddots
\end{array}\right],
$$

where $F_{n}$ is a shift with weights $\left\{a_{1}^{(n)}, a_{2}^{(n)}, \ldots\right\}, G_{n}=\operatorname{diag}\left\{b_{1}^{(n)}, b_{2}^{(n)}, \ldots\right\}\left(G_{n}\right.$ may have more rows than columns), and these entries satisfy:

(I) $\left(a_{j}^{(n)}\right)^{2}+\left(b_{j}^{(n)}\right)^{2}-\left(a_{j-1}^{(n)}\right)^{2} \geq 0\left(b_{j}^{(1)}=0\right.$ for all $\left.j\right)$,

(II) $b_{j}^{(n)}=0 \Rightarrow b_{j+1}^{(n)}=0$,

(III) there exists a constant $M$ such that $\left|a_{j}^{(n)}\right| \leq M$ and $\left|b_{j}^{(n)}\right| \leq M$ for $n=$ $2,3, \ldots$, and $j=1,2, \ldots$, where

$$
b_{j}^{(n+1)}=\left[\left(a_{j}^{(n)}\right)^{2}+\left(b_{j}^{(n)}\right)^{2}-\left(a_{j-1}^{(n)}\right)^{2}\right]^{1 / 2}
$$

and $a_{j}^{(n+1)}=a_{j}^{(n)} b_{j+1}^{(n+1)} / b_{j}^{(n+1)}$ (if $b_{j_{0}}^{(n)}=0$ then $a_{j_{0}}^{(n)}$ is taken to be zero).

Conditions (I), (II), and (III) were then shown to be sufficient as well. Interestingly, they can be reformulated in operator forms as follows:

(I') $D_{n} \geq 0$

(II') $F_{n} \operatorname{ker} D_{n} \subset \operatorname{ker} D_{n}$,

(III') $\left\|F_{n}\right\|,\left\|D_{n}\right\| \leq M$, where $G_{n+1}=D_{n}^{1 / 2}, D_{1}=F_{1}^{*} F_{1}-F_{1} F_{1}^{*}, D_{n+1}=$ $\left.D_{n}\right|_{H_{n+1}}+F_{n+1}^{*} F_{n+1}-F_{n+1} F_{n+1}^{*}, H_{n+1}=\left(\operatorname{Ran} D_{n}\right)^{-}$, and $F_{n+1}$ denotes the continuous extension of $D_{n}^{1 / 2} F_{n} D_{n}^{-1 / 2}$ to $\operatorname{Ran}\left(D_{n}^{1 / 2}\right)^{-}\left(=H_{n+1}\right)$ from $\operatorname{Ran}\left(D_{n}^{1 / 2}\right)$. Indeed, $\left(\mathrm{I}^{\prime}\right)$ and $\left(\mathrm{III}^{\prime}\right)$ are direct translation of (I) and (III), respectively. As for the equivalence of (II) and (II'), (II) implies (II') is obvious; to see the converse, it is enough to show that no shift $F_{n}$ (defined on $H_{n}$ ) has zero weights. But this can

Received by the editors December 15, 1986 and, in revised form, March 5, 1987.

1980 Mathematics Subject Classification (1985 Revision). Primary 47B20, 47B37.

Key words and phrases. Subnormal weighted shifts. 
be done by an induction argument. These three conditions happen to be precisely Spitkovskii's subnormality criterion [4].

Next we use this reformulation to show the subnormality of a class of shifts. In [1], C. Cowen and J. Long constructed a subnormal Toeplitz operator that is neither analytic nor normal and thus answered negatively a question raised by Halmos [2]. A key step in the construction is to show:

LEMMA [1]. If $0<\alpha<1$, the weighted shift with weights $w_{n}=\left(1-\alpha^{2 n+2}\right)^{1 / 2}$ for $n=0,1,2, \ldots$ is subnormal.

The proof of the lemma is based on the Berger-Gellar-Wallen criterion but involves some tricky special function theory; see reference in [1]. So it would be nice to have a purely operator-theoretic proof. In fact, it was $\mathrm{Ma}$ Ji Pu and Zhou Shao Jie [3] who first used the Spitkovskii's characteristion to prove the subnormality of the weighted shift with weights $w_{n}=\left(2-1 / 2^{n}\right)^{1 / 2}$ for $n=0,1,2, \ldots$ Since this shift is a constant multiple of the shift $(\alpha=1 / \sqrt{2})$ in the lemma, we thought it is probably not an isolated case. It is not, as the following computation shows: Let $F_{1}$ be a shift in the lemma. Then: $(\mathrm{I})^{\prime} D_{1}=F_{1}^{*} F_{1}-F_{1} F_{1}^{*}=\operatorname{diag}\left\{1-\alpha^{2}, \alpha^{2}-\alpha^{4}, \ldots\right\} \geq 0$ and $D_{n+1}=\left.D_{n}\right|_{H_{n+1}}+F_{n+1}^{*} F_{n+1}-F_{n+1} F_{n+1}^{*}=\left(1+\alpha^{2}+\cdots+\alpha^{2 n}\right) D_{1} \geq 0$,

(II) ${ }^{\prime} F_{n} \operatorname{ker} D_{n} \subset \operatorname{ker} D_{n}$ since $\operatorname{ker} D_{n}=\{0\}$,

(III) ${ }^{\prime} F_{n+1}=D_{n}^{1 / 2} F_{n} D_{n}^{-1 / 2}=\alpha^{n} F_{1}$ and $D_{n}$ are clearly bounded. Thus all three conditions are satisfied and so $F_{1}$ is subnormal.

\section{BIBLIOGRAPHY}

1. C. C. Cowen and J. J. Long, Some subnormal Toeplitz operators, J. Reine Angew. Math. 351 (1984), 216-220.

2. P. R. Halmos, Ten problems in Hilbert space, Buli. Amer. Math. Soc. 76 (1970), 887-933.

3. $\mathrm{Ma} \mathrm{Ji} \mathrm{Pu}$ and Zhou Shao Jie, A necessary and sufficient condition for an operator to be subnormal, Nanjing Daxue Xuebao 2 (1985), 258-267. (Chinese)

4. I. M. Spitkovskii, A criterion for normality of operators in Hilbert space, Funct. Anal. Appl. 16 (1982), 148-150.

5. J. G. Stampfli, Which weighted shifts are subnormal?, Pacific J. Math. 17 (1966), 367-379.

Department of Mathematics, Texas Christian University, Fort Worth, TEXAS 76129 